\title{
A COMPARISON THEOREM FOR CONJUGATE POINTS OF GENERAL SELFADJOINT DIFFERENTIAL EQUATIONS ${ }^{1}$
}

\section{KURT KREITH}

Abstract. Comparison theorems for conjugate points of fourth order selfadjoint differential equations have been established by $\mathrm{J}$. Barrett. In the present paper a more direct method of proof is used to generalize Barrett's theorem to general selfadjoint equations of even order while replacing certain pcintwise conditions on the coefficients by weaker integral conditions. Further generalizations are indicated to nonlinear equations, to differential inequalities, and to certain nonselfadjoint equations.

Let $u(x)$ and $v(x)$ be nontrivial solutions of

$$
\begin{aligned}
& \sum_{k=0}^{n}\left((-1)^{k} r_{k}(x) u^{(k)}(x)\right)^{(k)}=0 \text { and } \\
& \sum_{k=0}^{n}\left((-1)^{k} R_{k}(x) v^{(k)}(x)\right)^{(k)}=0
\end{aligned}
$$

respectively, where the coefficients of (1) and (2) are real valued and satisfy

$$
\begin{array}{lll}
r_{n}>0 & \text { and } \quad r_{k} \in C^{k}[\alpha, \infty) & \text { for } k=0, \cdots, n ; \\
R_{n}>0 & \text { and } \quad R_{k} \in C^{k}[\alpha, \infty) & \text { for } k=0, \cdots, n .
\end{array}
$$

We shall be interested in nontrivial solutions of (1) and (2) which satisfy

$$
\begin{aligned}
u(\alpha) & =u^{\prime}(\alpha)=\cdots=u^{(n-1)}(\alpha)=0 \\
& =u(\beta)=u^{\prime}(\beta)=\cdots=u^{(n-1)}(\beta), \\
v(\alpha) & =v^{\prime}(\alpha)=\cdots=v^{(n-1)}(\alpha)=0 \\
& =v(\gamma)=v^{\prime}(\gamma)=\cdots=v^{(n-1)}(\gamma) .
\end{aligned}
$$

The smallest number $\beta>\alpha$ such that (3) is satisfied nontrivially by a solution of (1) is denoted by $\mu_{1}(\alpha)$ and called the first conjugate point of $x=a ; \eta_{1}(\alpha)$ is similarly defined by (2) and (4). The purpose of this

Received by the editors September 8, 1969.

AMS Subject Classifications. Primary 3442; Secondary 3420.

Key Words and Phrases. Conjugate points, matrix system, fundamental system of solutions.

1 Research supported by National Science Foundation Grant GP-11219. 
paper is to establish relations among the coefficients of (1) and (2) which assure that $\eta_{1}(\alpha) \leqq \mu_{1}(\alpha)$.

Oscillation properties of higher order selfadjoint differential equations have recently been studied by several authors who reduce such equations to first order vector systems. For example, Barrett [1] reduces fourth order equations to vector systems of the form

$$
\boldsymbol{u}^{\prime}=A \boldsymbol{u}+B \boldsymbol{w}, \quad \mathbf{w}^{\prime}=C \boldsymbol{u}+A \boldsymbol{w}
$$

where $A, B$, and $C$ are nonsymmetric $2 \times 2$ matrices. Hunt [2] and Howard [3] reduce equations of order $2 n$ to vector systems of the form

$$
\boldsymbol{u}^{\prime}=A \boldsymbol{u}+B \boldsymbol{w}, \quad \mathbf{w}^{\prime}=C \boldsymbol{u}-A^{*} w,
$$

where $B$ and $C$ are symmetric matrices. In all cases, the authors then use a device due to Sternberg [4] to reduce these vector systems to the form

$$
\tilde{u}^{\prime}=E \tilde{w}, \quad \tilde{w}^{\prime}=-F \tilde{u}
$$

where $E$ and $F$ are symmetric. The system (7) is then studied by means of matrix Riccati equations or other techniques.

In this paper we shall show that an identity established by the author [5] for systems of the form (7) can also be applied to the more general systems of the form (6). This fact allows us to circumvent the transformation of Sternberg and to establish extremely simple comparison criteria for conjugate points of (1) and (2).

Given solutions $u(x)$ and $v(x)$ of (1) and (2), respectively, we define

$$
\begin{aligned}
& u(x)=\left(\begin{array}{l}
u \\
u^{\prime} \\
\vdots \\
\dot{u}^{(n-1)}
\end{array}\right), \quad v(x)=\left(\begin{array}{l}
v \\
v^{\prime} \\
\vdots \\
v^{(n-1)}
\end{array}\right) \text {, } \\
& w=\left(\begin{array}{l}
-\left[(-1)^{n}\left(r_{n} u^{(n)}\right)^{(n-1)}+(-1)^{n-1}\left(r_{n-1} u^{(n-1)}\right)^{(n-2)}+\cdots-r_{1} u^{\prime}\right] \\
{\left[(-1)^{n}\left(r_{n} u^{(n)}\right)^{(n-2)}+\cdots+r_{2} u^{\prime \prime}\right]} \\
\vdots \\
{\left[r_{n} u^{(n)}\right]}
\end{array}\right) \text {, } \\
& z=\left(\begin{array}{l}
-\left[(-1)^{n}\left(R_{n} v^{(n)}\right)^{(n-1)}+(-1)^{n-1}\left(R_{n-1} v^{(n-1)}\right)^{(n-2)}+\cdots-R_{1} v^{\prime}\right] \\
{\left[(-1)^{n}\left(R_{n} v^{(n)}\right)^{(n-2)}+\cdots+R_{2} v^{\prime \prime}\right]} \\
\vdots \\
{\left[R_{n} v^{(n)}\right]}
\end{array}\right) .
\end{aligned}
$$


Equations (1) and (2) can then be written as vector systems of the form

$$
\begin{array}{ll}
\boldsymbol{u}^{\prime}=a \boldsymbol{u}+b \boldsymbol{w}, & \boldsymbol{w}^{\prime}=c \boldsymbol{u}-a^{*} w, \\
\boldsymbol{v}^{\prime}=A \boldsymbol{v}+B \boldsymbol{z}, & \boldsymbol{z}^{\prime}=C \boldsymbol{v}-A^{*} \boldsymbol{z},
\end{array}
$$

where

(i) $A=a=\left(a_{i j}\right)$ and $a_{i j}=1$ if $j=i+1$

$$
=0 \text { if } j \neq i+1 \text {, }
$$

(ii) $b=\operatorname{diag}\left(0,0, \cdots, 1 / r_{n}\right) ; B=\operatorname{diag}\left(0,0, \cdots, 1 / R_{n}\right)$,

(iii) $c=\operatorname{diag}\left(r_{0}, r_{1}, \cdots, r_{n-1}\right) ; C=\operatorname{diag}\left(R_{0}, R_{1}, \cdots, R_{n-1}\right)$.

In addition to $\left(2^{\prime}\right)$ we shall be interested in studying the matrix system

$$
V^{\prime}=A V+B Z, \quad Z^{\prime}=C V-A^{*} Z .
$$

For if we consider a fundamental system of solutions $v_{1}, \cdots, v_{n}$ for (2) which satisfies

$$
v_{i}^{(j-1)}(\alpha)=0, \quad i, j=1, \cdots, n ; \quad v_{i, k}^{(j-1)}(\alpha)=\delta_{i j}, \quad j=1, \cdots, n
$$

where

$$
v_{i, k}=\sum_{s=0}^{k-1}\left((-1)^{n-s} R_{n-s} v_{i}^{(n-s)}\right)^{(k-1-8)}, \quad k=1, \cdots, n,
$$

then the column vectors

(8) $v_{i}(x)=\left(\begin{array}{l}v_{i} \\ v_{i}^{\prime} \\ \vdots \\ \dot{v}_{i}^{(n-1)}\end{array}\right), z_{i}(x)=\left(\begin{array}{c}-\left[(-1)^{n}\left(R_{n} v_{i}^{(n)}\right)^{(n-1)}+\cdots-R_{1} v_{i}^{\prime}\right] \\ {\left[(-1)^{n}\left(R_{n} v_{i}^{(n)}\right)^{(n-2)}+\cdots+R_{2} v_{i}^{\prime \prime}\right]} \\ \vdots \\ {\left[\dot{R}_{n} v_{i}^{(n)}\right]}\end{array}\right)$

satisfy $\left(2^{\prime}\right)$ for $i=1, \cdots, n$, and the matrices $V(x), Z(x)$ whose columns are $v_{i}$ and $z_{i}$, respectively, satisfy $\left(2^{\prime \prime}\right)$. Furthermore it follows readily [1], [3], that $\eta_{1}(\alpha)$ is the smallest $\gamma>\alpha$ for which $\operatorname{det} V(\gamma)=0$.

In connection with the system $\left(2^{\prime \prime}\right)$, it is also important to note that if $B$ and $C$ are symmetric, then $d\left[V^{*} Z-Z^{*} V\right] / d x=0$, so that $V(\alpha)=0$ implies that $V^{*} Z=Z^{*} V$. If, in addition, $V^{-1}$ exists, then

$$
Z V^{-1}=V^{*-1} Z^{*}=\left(Z V^{-1}\right)^{*} \text {. }
$$

Finally, we shall require the following notion of ordering among matrices.

Definition. Given matrices $P$ and $Q$, we shall write $Q<P$ in case

(i) the range of $Q$ is orthogonal to the null space of $P$ and 
(ii) $Q^{*}-Q^{*} P^{-1} Q$ is positive semidefinite, where $P^{-1}$ is to be interpreted as the inverse of $P$ in the range of $Q$.

Lemma. Let $u(x), w(x)$ be a nontrivial vector solution of $\left(1^{\prime}\right)$ and let $V(x), Z(x)$ be a matrix solution of $\left(2^{\prime \prime}\right)$, where $a=A, B$ is symmetric and positive semidefinite, and $b \prec B$. If det $V(x) \neq 0$ and (9) is satisfied, then

$$
\begin{aligned}
d\left[\mathbf{u}^{*} \boldsymbol{w}-\mathbf{u}^{*} Z V^{-1} \mathbf{u}\right] / d x & =\mathbf{u}^{*}(c-C) \boldsymbol{u} \\
& +\mathbf{w}^{*}\left(b^{*}-b^{*} B^{-1} b\right) \boldsymbol{w}+\boldsymbol{y}^{*} B \boldsymbol{y},
\end{aligned}
$$

where $y=B^{-1} b w-Z V^{-1} u$.

Proof. Expanding the left side of (10) yields

$$
\begin{aligned}
d\left[\mathbf{u}^{*} w-\mathbf{u}^{*} Z V^{-1} \mathbf{u}\right] / d x= & \mathbf{u}^{*} \boldsymbol{w}^{\prime}-\mathbf{u}^{*} Z^{\prime} V^{-1} \mathbf{u}+\mathbf{u}^{* \prime} \boldsymbol{w} \\
& -\boldsymbol{u}^{* \prime} Z V^{-1} \boldsymbol{u}-\mathbf{u}^{*} Z V^{-1} \boldsymbol{u}^{\prime}+\mathbf{u}^{*} Z V^{-1} V^{\prime} V^{-1} \mathbf{u} .
\end{aligned}
$$

Using the differential equations $\left(1^{\prime}\right)$ and $\left(2^{\prime \prime}\right)$ we get

$$
\begin{aligned}
d\left[\mathbf{u}^{*} w-\mathbf{u}^{*} Z V^{-1} \mathbf{u}\right] / d x & =\mathbf{u}^{*}\left(c \boldsymbol{u}-a^{*} w\right)-\mathbf{u}^{*}\left(C V-A^{*} Z\right) V^{-1} \mathbf{u} \\
+ & \left(\boldsymbol{u}^{*} a^{*}+w^{*} b^{*}\right) \boldsymbol{w}-\left(\mathbf{u}^{*} a^{*}+w^{*} b^{*}\right) Z V^{-1} \mathbf{u} \\
& -\mathbf{u}^{*} Z V^{-1}(a \boldsymbol{u}+b \boldsymbol{w})+\mathbf{u}^{*} Z V^{-1}(A V+B Z) V^{-1} \mathbf{u} .
\end{aligned}
$$

Cancelling the terms of the form $u^{*} a w, u^{*} A^{*} Z V^{-1} u, u^{*} Z V^{-1} a u$, inserting $-w^{*} b^{*} B^{-1} b w+w^{*} b^{*} B^{-1} b w$, and rearranging terms yields

$$
\begin{aligned}
d\left[\mathbf{u}^{*} \boldsymbol{w}-\mathbf{u}^{*} Z \boldsymbol{V}^{-1} \boldsymbol{u}\right] / d x=\boldsymbol{u}^{*}(c-C) \boldsymbol{u}+\mathbf{w}^{*}\left(b^{*}-b^{*} B^{-1} b\right) \boldsymbol{w} \\
+\left[\left(B^{-1} b \boldsymbol{w}\right)^{*}-\left(Z V^{-1} \boldsymbol{u}\right)^{*}\right] B\left[\left(B^{-1} b \boldsymbol{w}\right)-\left(Z V^{-1} \boldsymbol{u}\right)\right] .
\end{aligned}
$$

In this last manipulation we have made use of the assumption $B=B^{*}$ and of (9). The identity (10) is clearly equivalent to the last equation above.

THEOREM. Let $u(x)$ be a nontrivial solution of (1) satisfying (3), where $\beta=\mu_{1}(\alpha)$. If

(i) $0<R_{n}(x) \leqq r_{n}(x)$ for $\alpha \leqq x \leqq \beta$ and

(ii) $R_{k}(x) \leqq r_{k}(x)$ for $\alpha \leqq x \leqq \beta$ and $k=0, \cdots, n-1$, then $\eta_{1}(\alpha) \leqq \beta$.

Proof. Referring back to the definitions of $a, A, b, B, c$, and $C$, in the systems $\left(1^{\prime}\right)$ and $\left(2^{\prime}\right)$, it is clear that hypothesis (i) implies that $b \prec B$. Suppose now that $\eta_{1}(\alpha)>\beta$ so that the matrix $V$, whose columns are given by (8), satisfies det $V(x) \neq 0$ for $\alpha<x \leqq \beta$. By continuity we may replace the point $\alpha$ used to define $V$ by a slightly smaller $\alpha^{\prime}<\alpha$ and thereby assure $V(x) \neq 0$ for $\alpha \leqq x \leqq \beta$. Since $V\left(\alpha^{\prime}\right)=0,(9)$ is satisfied and by the lemma above (10) holds for $\alpha \leqq x \leqq \beta$. Since $B$ is positive semidefinite, 
(11)

$$
d\left[\mathbf{u}^{*} \mathbf{w}-\mathbf{u}^{*} Z V^{-1} \mathbf{u}\right] / d x \geqq \mathbf{u}^{*}(c-C) \mathbf{u}+\mathbf{w}^{*}\left(b^{*}-b^{*} B^{-1} b\right) \boldsymbol{w}
$$

with equality iff $y^{*} B y=0$. However this last condition is satisfied iff

$$
B^{-1} b w-B^{-1} B Z V^{-1} u=0 .
$$

Since $B^{-1}$ is nonsingular on the range of $b,(12)$ is satisfied iff

$$
\begin{gathered}
b w-B Z V^{-1} u=0, \quad u^{\prime}-a u-\left(V^{\prime}-A V\right) V^{-1} u=0, \\
u^{\prime}-V^{\prime} V^{-1} u=0
\end{gathered}
$$

or finally iff

$$
\left(V^{-1} u\right)^{\prime}=0
$$

which implies that $\boldsymbol{u}=V \boldsymbol{k}$ for some constant vector $\boldsymbol{k}$ and that $V(\alpha)=V(\beta)=0$. Ruling out the contradictory case $y^{*} B y \equiv 0$, we integrate both sides of (11) from $\alpha$ to $\beta$ to get

$$
0>\int_{\alpha}^{\beta}\left[\mathbf{u}^{*}(c-C) u+w^{*}\left(b^{*}-b^{*} B^{-1} b\right) w\right] d x .
$$

However our hypotheses (i) and (ii) assure that $c-C$ and $b^{*}-b^{*} B^{-1} b$ are positive semidefinite, and therefore the right side of (13) must be nonnegative. This yields the desired contradiction and shows that $\eta_{1}(\alpha) \leqq \beta$.

In case $n=2$, the above theorem reduces to a result of Barrett [1, Theorem 5.1]. While Barrett's proof would also seem to allow generalization to arbitrary $n$, the present techniques are substantially more direct and less laborious. Furthermore the present method of proof allows a number of generalizations of the theorem which are sketched below.

1. Hypotheses (i) and (ii) can clearly be replaced by weaker integral conditions which assure that the right side of (13) is nonnegative. If $u(x)$ is a solution of (1) which realizes the conjugate points $\alpha, \beta$, then this integral condition becomes

$$
\int_{\alpha}^{\beta} \sum_{k=0}^{n}\left(r_{k}-R_{k}\right)\left[u^{(k)}\right]^{2} d x \geqq 0 .
$$

2. In the proof of the lemma, no assumptions need be made regarding the linearity of the matrices appearing in $\left(1^{\prime}\right)$ and $\left(2^{\prime \prime}\right)$. Accordingly the coefficients of (1) and (2) may take the form $r_{k}\left(x, u, \cdots, u^{(n-1)}\right)$ and $R_{k}\left(x, v, \cdots, v^{(n-1)}\right)$ as long as the hypotheses of the theorem hold for $\alpha \leqq x \leqq \beta$ and for all values of the other arguments of the $r_{k}$ and $R_{k}$.

3. In case $\left(1^{\prime}\right)$ is replaced by the system 


$$
u^{\prime}=a u+b w, \quad u^{*} w^{\prime} \geqq u^{*} c u-u^{*} a^{*} w,
$$

the lemma yields an inequality in place of (10) which suffices to prove the theorem. As a result, we may replace the equation (1) by the inequality

$$
u \sum_{k=0}^{n}\left((-1)^{k} r_{k}(x) u^{(k)}(x)\right)^{(k)} \geqq 0 .
$$

4. In the proof of the lemma, no assumption is made regarding the symmetry of the matrices $b$ or $c$. Accordingly we may consider certain nonselfadjoint equations in place of (1). Defining

$$
\begin{aligned}
u_{n} & =r_{n} u^{(n)}, \\
u_{n-1} & =-u_{n}^{\prime}+r_{n-1} u^{(n-1)}, \\
u_{n-k} & =-u_{n-k+1}^{\prime}+q_{n-k} u^{(n-k+1)}+r_{n-k} u^{(n-k)}, \quad k=2, \cdots, n-1,
\end{aligned}
$$

we consider in place of (1) the equation

$$
u_{1}^{\prime}-q_{0} u^{\prime}-r_{0} u=0 .
$$

This equation can still be written in the form $\left(1^{\prime}\right)$ with $a$ and $b$ unchanged and

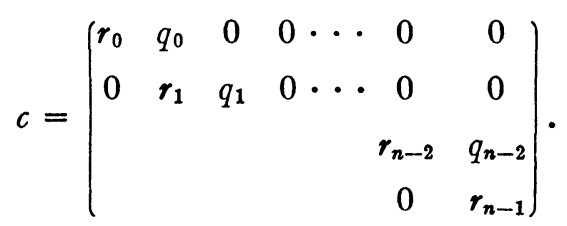

The comparison theorem remains valid if $c$ is replaced by the above matrix.

\section{REFERENCES}

1. J. H. Barrett, Fourth order boundary value problems and comparison theorems, Canad. J. Math. 13 (1961), 625-638. MR 24 \#A3350.

2. R. Hunt, The behavior of solutions of ordinary, self-adjoint differential equations of arbitrary even order, Pacific J. Math. 12 (1962), 945-961. MR 26 \#5239.

3. H. C. Howard, Some oscillation criteria for general self-adjoint differential equations, J. London Math. Soc. 43 (1968), 401-406. MR 37 \#3104.

4. R. L. Sternberg, Variational methods and non-oscillation theorems for systems of differential equations, Duke Math. J. 19 (1952), 311-322. MR 14, 50.

5. K. Kreith, A Picone identity for first order differential systems, J. Math. Anal. Appl. (to appear).

University of California, Davis, California 95616 